\title{
Erratum to: The Possibility of Harmless Destruction of an Asteroid Threatening the Earth
}

\author{
A. G. Aleksandrova ${ }^{1}$, T. Yu. Galushina ${ }^{1}$, A. B. Prishchepenko ${ }^{2}$, \\ K. V. Kholshevnikov*3,4, and V. M. Chechetkin ${ }^{5,6}$ \\ ${ }^{1}$ Tomsk State University, Tomsk, Russia \\ 2"Sirius" Scientific Research and Experimental Center, Moscow, Russia \\ ${ }^{3}$ St. Petersburg State University, St. Petersburg, Russia \\ ${ }^{4}$ Institute of Applied Astronomy, Russian Academy of Sciences, St. Petersburg, Russia \\ ${ }^{5}$ Keldysh Institute of Applied Mathematics, Russian Academy of Sciences, Moscow, 125047 Russia \\ ${ }^{6}$ Kurchatov Research Institute, Moscow, 123182 Russia \\ Received November 27, 2019; revised November 27, 2019; accepted November 27, 2019
}

DOI: $10.1134 / \mathrm{S} 1063772919140014$

Page 786, FUNDING should read as follows:

This work was supported by the Russian Science Foundation (grant 18-12-00050) and the Program for Enhancing the Competitiveness of Tomsk State University.

The original article can be found online at https://doi.org/10.1134/S1063772919090014

\footnotetext{
*E-mail:kvk@astro.spbu.ru
} 